\title{
Bile acid malabsorption: a concise review
}

\begin{abstract}
Bile acid malabsorption (BAM) is a frequently unrecognized and misdiagnosed cause of chronic diarrhea. It is characterized by the disruption of normal enterohepatic circulation modifying bile acids (BAs) production, reabsorption and/or transportation. The level in which the abnormality appears gives BAM classification. Type 1 BAM is accounted to small bowel dysfunction, Type 2 to a primary affection or idiopathic, Type 3 to other gastrointestinal disorders and a fourth type may be related to hepatic BAs hyperproduction. BAM predominant symptom is chronic watery diarrhea. The 75 selenium homotaurocholic acid taurine (SeHCAT) retention test is considered the gold standard but it has a lot of practice limitations. The serum $7 \alpha$-hydroxy-4-cholesten-3-one (C4) concentration is next in line due to its simplicity and high sensitivity and specificity. Treatment is based on bile acid sequestrants.
\end{abstract}

Volume 4 Issue 2 - 2016

\author{
Jose M Nieto,' Amanda Bastidas ${ }^{2}$ \\ 'Borland Groover Clinic,Advanced Therapeutic Endoscopy \\ Center, USA
}

${ }^{2}$ Universidad Especialidades Espiritu Santo, Ecuador

Correspondence: Jose M Nieto, Assistant Professor of Medicine, UCF School of Medicine Professor of Medicine, UEES, Ecuador 4800 Belfort Rd, second floor Jacksonville, FL 32256, USA, Email drjnieto@gmail.com

Received: November 01, 2015 | Published: February 10, 2016

Keywords: bile acid malabsorption; chronic diarrhea; bile acid sequestrants

Abbreviations: BAM, bile acid malabsorption; CA, cholic acid; CDCA, chenodeoxycholic acid; DCA, deoxycholic acid; ASBT, apical sodium-dependent bile acid transporter; IBS-D, diarrhea-predominant irritable bowel syndrome; SeHCAT, selenium homotaurocholic acid test; FGF, fibroblast growth factor; GPBAR1, G protein-coupled bile acid receptor 1; CYP7A1, cholesterol 7ahydroxylase; GCMS, gas chromatography-mass spectrometry; LC-MS/MS, liquid chromatography-tandem mass spectrometry; HPLC-MS, high performance liquid chromatography-mass spectrometry

\section{Introduction}

\section{Definition}

Bile acid malabsorption (BAM) is a disorder resulting from dysregulation of the enterohepatic recycling of bile acids and of bile acid production, ${ }^{1}$ is often regarded as a rare phenomenon, reflected in a survey of gastroenterologist in the United Kingdom, ${ }^{2}$ the most common clinical presentation is chronic diarrhea, approximately onethird of new patients presented this symptom.

\section{Normal enterohepatic circulation}

The enterohepatic circulation was first described by Small et al. ${ }^{3}$ four decades ago (Figure 1). The primary bile acids are cholic acid (CA) and chenodeoxycholic acid (CDCA); they are synthesized from cholesterol in the liver, conjugated with glycine or taurine to increase their water solubility and secreted to bile. Secondary bile acid are deoxycholic acid (DCA) and lithocholic acid, they are derived from primary bile acids as a result of modifications such as deconjugation, 7-dehydroxylation by intestinal bacteria, this causes de increase passive absorption of secondary bile acids in the colon. ${ }^{1}$ Approximately $95 \%$ of primary bile acids are reabsorbed by the distal ileum through active uptake by the apical sodium-dependent bile acid transporter (ASBT), returned to the liver via the portal circulation and taken up by hepatocytes. A small percentage of bile acids entering the colon can be passively absorbed, resulting in an overall net loss of $1 \%$ to $3 \% .{ }^{4}$ The conservation of the bile acid pool is altered by more rapid intestinal transit and changes in gut flora because of the different types of dieting, medications or other factors. Approximately $0.5 \mathrm{~g}$ of bile acids are synthesized in the liver per day, ${ }^{5}$ the total bile acid pool is approximately $3 \mathrm{~g}$, of which $95 \%$ is reclaimed from the ileum and a small amount of unconjugated bile acids is recaptured in the colon via passive non ionic diffusion. ${ }^{6,7}$ The cycle repeats for almost 4 to 12 times per day, resulting in an estimated loss of $0.2 \mathrm{~g} /$ day to $0.6 \mathrm{~g} / \mathrm{day}$ in the feces, the amount lost must be replaced with bile acid synthesized by the hepatocytes. ${ }^{5}$

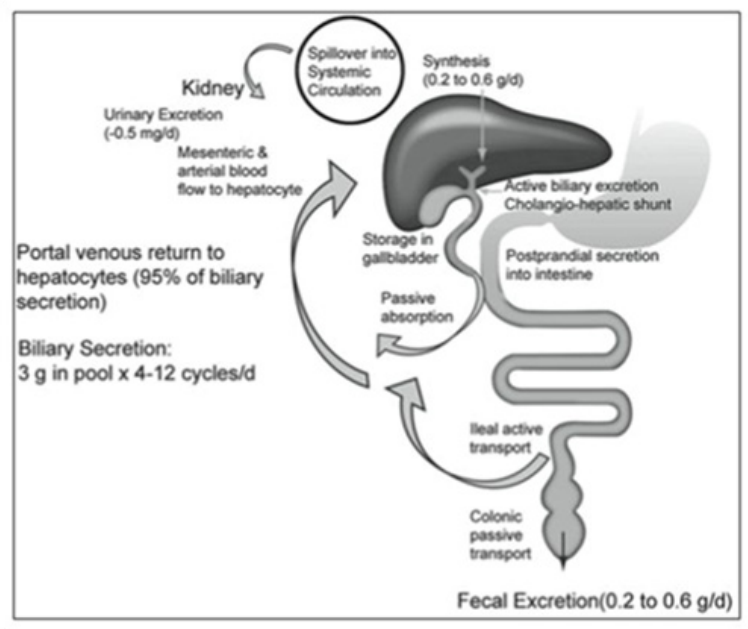

Figure I Enterohepatic circulation of bile acids. ${ }^{6}$

\section{Background}

BAM is a common, under-recognized, cause of chronic diarrhea with an estimated prevalence of $4 \%$ to $5 \%$ and $>90 \%$ in patients with resected Crohn's disease, $11 \%$ to $52 \%$ of unresected Crohn disease patients and $33 \%$ in diarrhea-predominant irritable bowel syndrome patients. ${ }^{1}$ For almost 30 years, a series of studies has suggested that BAM is frequently not considered as a possible diagnosis. ${ }^{2}$ The present review summarizes recent developments in the etiology, epidemiology, clinical features, diagnosis and treatment of BAM.

\section{Etiology and epidemiology}

\section{Classification of bile acid malabsorption}

The causes of BA diarrhea are based on the original classification of BAM: ${ }^{3}$ 
Type 1: Ileal dysfunction and impaired reabsorption such as Crohn's disease.

Type 2: Primary, or idiopathic, BAM produces a similar picture of increased fecal BAs, watery diarrhea, and response to BA sequestrants in the absence of ileal or other obvious gastrointestinal disease.

Type 3: Other gastrointestinal disorders such as small intestinal bacteria overgrowth, celiac disease, or chronic pancreatitis.

A fourth category of BAM may result from excessive hepatic BA synthesis, this disorder origins from the use of hypoglycemic drugs (metformin).

\section{Association with diseases}

In order to treat BAM and bile acid diarrhea, the diagnosis has to be clinically suspected, however, it is a diagnosis that is often forgotten by the majority of gastroenterologists. As mentioned before with its classification, several conditions are associated with BAM.

The normal enterohepatic circulation of bile salts is altered most of the time by ileal disease. Following ileal resection, typically due to Crohn's disease, Bas are not absorbed normally, which leads to BAM. A complication of Crohn's ileitis without resection can result in alteration of bile acid absorption. These conditions, related to ileal dysfunction, were called Type 1 BAM, with the idiopathic condition, classified as Type 2 BAM.

Several other gastrointestinal conditions are related to the interference of the normal physiology of bile acid reabsorption; these were classified as Type 3 BAM and include conditions such as pancreatic insufficiency, cholecystectomy and small intestinal bacterial overgrowth. Often the gastroenterologists don't recognize BAM in patients who are treated for malignant tumors and may have had radiation ileitis, pancreatitis, resection and bacterial overgrowth.

BAM should be considered in patients presenting the aforementioned conditions since it is highly associated to them and should not stay as an under recognized diagnosis.

\section{US and worldwide data}

Even though BAM is usually seen in patients with ileal disease, $25 \%$ to $50 \%$ of patients with functional diarrhea or diarrheapredominant irritable bowel syndrome (IBS-D), show some evidence of BAM. It has been estimated that at least $1 \%$ of the population in Western Countries are affected by this condition.

The use of new diagnostic methods, such as the Selenium HomotauroCholic Acid Test (SeHCAT), has allowed researchers to estimate the prevalence of BAM and diarrhea. BAM secondary to ileal disease is common. A retrospective review of 298 patients with chronic diarrhea found positive test results in 15 of 29 patients with unresected Crohn's disease (51.7\%), 40 of 43 with resected Crohn's disease (93\%), 12 of 12 patients after small bowel resection (100\%) and 2 of 3 patients following radiation injuries $(66.7 \%)$.

As for type 2 BAM, the estimated prevalence ranges from $37.5 \%$ to $59.6 \%$. One-third of patients with a diagnosis of IBS-D report BAM. A review of 18 studies (1223 patients) reported that $10 \%$ of patients presented severe BAM, $32 \%$ moderate BAM, and $26 \%$ reported some degree of BAM (mild, moderate or severe).

Finally, type 3 BAM is not uncommon in patients with an array of gastrointestinal pathologies that are not related to ileal dysfunction. BAM is reportedly associated to altered bicarbonate secretion in chronic pancreatitis. In celiac disease, BAM is caused by impairments in gall bladder motor function and atrophy of the small intestinal mucosa.

\section{Clinical features and findings}

Bile acid malabsorption usually manifests as a chronic, watery diarrhea. In type 2 BAM this can be the only symptom the patient refers, but gastroenterologists often do not relate the condition as a possible cause for chronic diarrhea. BAM should be clinical suspected and investigated in the presence of chronic diarrhea following a resection in Crohn's disease or associated to other conditions such as pancreatitis.

\section{Review of physiopathologic and clinical links between diseases}

Although it is known that type 1 BAM is caused by ileal disease and type 3 BAM by a variety of gastrointestinal conditions, the mechanisms for the idiopathic BAM (type 2) are still not entirely known. Recent literature has proposed several theories to explain the pathophysiology of type 2 BAM:

a. Defective feedback inhibition of bile acid biosynthesis by FGF19: FGF-19 is produced in the ileum to regulate hepatic BA synthesis, in response to their absorption. Walters et al. ${ }^{8}$ reported that in patients with type 2 BAM, the levels of FGF-10 in serum are lower.

b. Genetic mutations in the apical sodium-bile acid transporter (ASBT): These mutations are extremely rare and have been excluded as a cause of idiopathic BAM.

c. Accelerated small bowel transit bypassing active bile acid transport in the ileum: This has been hypothesized as a cause of type 2 BAM, but while it is possible, the apical sodium-bile acid transporter's affinity for BAs to make it an unlikely cause.

d. Genetic variations in the proteins involved in feedback regulation of bile acid synthesis, KlothoB gene and fibroblast growth factor 4 gene: This theory is based on the association of SNP rs17618244 in the KlothoB gene with colonic transit in IBS-D. Pharmacogenetic studies suggest that variations in the KlothoB gene may result in bowel response to BAM.

e. Upregulation of the membrane bound bile acid receptor (GPBAR1): GPBAR1 is a member of the G protein-coupled receptor family that works as a surface receptor for BA. Studies show that variations in GPBAR1 may predispose to quantitative changes in the excretion of BA.

\section{Diagnosis}

\section{Laboratory studies}

There are multiple studies that can be requested to evaluate the presence of BAM in a patient. The four main diagnostic tests that can prove the existence of BAM are $\mathrm{C}$-glycocholate breath and stool test, Selenium HomotauroCholic Acid Test (SeHCAT), serum 7 $\alpha$-hydroxy-4-cholesten-3-one (C4) and measurement of bile acids in feces. A surge of BAs in feces is the most reliable way to prove the malabsorption, but fecal tests are difficult to perform and mostly done in a few research centers, so more practical alternatives were sought in SeHCAT and C4.

\section{C-glycocholate breath and stool test}

This diagnostic method is capable of determining bacterial dependent deconjugation that happens in the digestive tract due to BAM or other causes. 
The C-glycocholate solution is administered orally and mixes with the pool of BAs. Bacteria in the gastrointestinal tract sever the bond between glycine and cholic acid. C-glycine is released, metabolized and the result is exhaled as $\mathrm{CO} 2$. If early breath excretion of $\mathrm{CO} 2$ is detected, this is highly suggestive of bacterial overgrowth in the small intestine. If not, it is inferred that the solution entered the large intestine and will be metabolized by colonic bacteria. A small part is excreted in stool. Hence, a stool collection after the breath test can detect BAM.

The methodology used for this test is complex and laborious. After the C-glycocholate is ingested in a meal, exhaled air is blown every hour for at least 6 hours to measure CO2. Stool is collected for 24 hours to also measure $\mathrm{CO} 2$. An estimate of fecal bile acid excretion is provided. Reference range interpretive values varied between study centers.

Even though the C-glycocholate breath and stool test provided a reliable way to diagnose BAM, it also presented several disadvantages. First, the amount of $\mathrm{CO} 2$ exhaled during the first hours didn't always differentiate deconjugation happening in the various parts of the digestive tract, so a stool test was usually obligatory. Also, there were concerns over radiation and the taxing nature of the test. These disadvantages and the development of more practical and improved diagnostic tests, have led to the decision of many centers to discontinue the $\mathrm{C}$-glycocholate breath and stool test.

Selenium homotaurocholic acid test (SeHCAT): The SeHCAT is the most commonly used test for diagnosis of BAM. The test uses a synthetic BA (selenium homotaurocholic) that resists passive diffusion and bacterial degradation. The bile acid is administered orally via capsule and the retention is measured through a gamma camera after a week. A retention value of less than $10 \%$ is considered abnormal and indicates BAM. The test has sensitivity for BAM of 80 $90 \%$ and specificity of $70-100 \%$.

The main advantange that this diagnostic test offers is the avoidance of stool collections, which makes it less laborious and also improves patient compliance. It also provides a measurement of severity of the BAM. Other advantages include a decrease in the radiation dose that extra-abdominal organs may receive, isotope distribution is not affected by body build or position and it provides less borderline results and false negatives when compared with the C-glycocholate test. One disadvantage is that this test is not available in every center and it is not approved in some countries.

The interpretation of the results of the SeHCAT is simple. Retention at 7 days of $\geq 15 \%$ BA is said to be normal, $10-15 \%$ is considered mild BAM, $5-10 \%$ moderate and $<5 \%$, severe.

\section{Serum $7 \alpha$-hydroxy-4-cholesten-3-one (C4)}

C4 was first developed to measure BA synthesis and the removal of LDL of circulation in clinical trials that researched ways to reduce cardiovascular risk. In humans, BA synthesis is regulated by the enzyme cholesterol 7ahydroxylase (CYP7A1). C4 is a product of CYP7A1 and its relation with BA synthesis has been checked by multiple studies.

Unlike the diagnostic tests previously mentioned, which require investing time and special equipment, serum $\mathrm{C} 4$ consists of a simple blood test. The test requires drawing blood in the morning after an overnight fasting, followed by isolation of $\mathrm{C} 4$ through liquid chromatography-tandem mass spectrometry. $\mathrm{C} 4$ is reported as serum concentration $(\mathrm{ng} / \mathrm{mL})$. An elevated serum $\mathrm{C} 4$ concentration is $>60.7 \mathrm{ng} / \mathrm{mL}$, however, this can vary with each center.
The C4 test has a high sensitivity and specificity (90 and 79\%) and negative and positive predictive values of $98 \%$ and $74 \%$, respectively, in comparison to the SeHCAT test. It has no relation to gender, age or serum cholesterol and it is specially useful in the screening of pediatric population since it doesn't expose them to radiation and it is relatively simple to obtain a sample.

\section{Fecal BA}

The diagnostic tests mentioned above are considered indirect, since they measure BA synthesis or retention. It is now possible to directly quantify fecal total and individual BA. Increased total fecal BA is reported in chronic diarrhea and in irritable bowel syndrome that presents with diarrhea (Figure 2). Determining fecal BA is an arduous technique and needs the expertise and skill of specialized technicians. Even though in the future it may become the gold standard test, it is not available in every center.

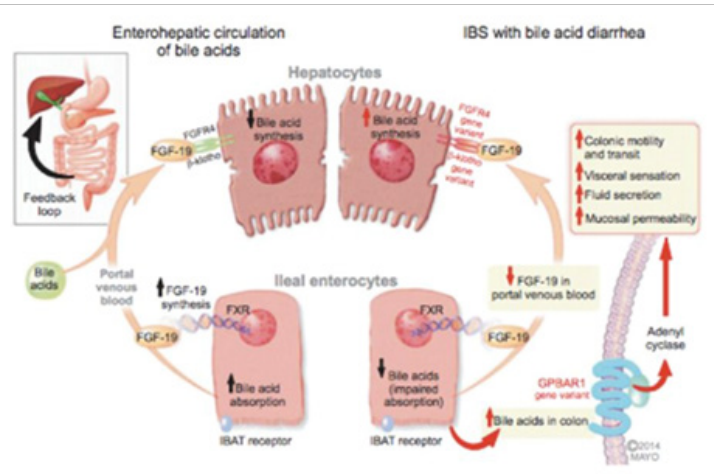

Figure 2 Mechanisms of bile acid (BA)-related bowel dysfunction in irritable bowel syndrome with diarrhea or idiopathic BA diarrhea. ${ }^{4}$

To perform the test, stool is collected during 48 hours after a high fat intake diet. Fecal BA can be measured through two assays: enzymatic and chromatographic. The enzymatic assay uses a NAD+ dependent steroid dehydrogenase enzyme to oxidize deconjugated BAs and produce NADH. The NADH is then converted to a product that can be measured and detected more easily. As for the chromatographic assay, the stool sample is weighed and frozen at $-25 \mathrm{oC}$ until analysis. The quantification can be performed with 3 methods: gas chromatography-mass spectrometry (GC-MS), liquid chromatography-tandem mass spectrometry (LC-MS/MS), or high performance liquid chromatography-mass spectrometry (HPLC-MS).

The main disadvantage of measuring fecal BAs is that it needs trained personnel and it is a complex process. Also, this test does not take into account the variation in daily BA excretion. Due to this variability, more than one stool sample is needed to achieve an accurate diagnosis of BAM.

\section{Treatment}

\section{Bile acid sequestrants}

The bile acid sequestrants, or binders, are considered the first line treatment for bile acid malabsorption and diarrhea. These drugs are administered orally and act by mopping up free bile acids in the small bowel and by preventing the secretory action bile acids in the large intestine. Presently, there are a few bile acid binders which are: cholestyramine, colestipol and colesevelam. All of them have shown effectiveness controlling bile acid-induced diarrhea. The dose can be titrated down or up, depending upon the patient's response. Higher doses can lead to constipation, but since they're not absorbed in the 
intestine, they don't have systemic side effects. Also, they have a lowering effect in the patient's cholesterol.

Cholestyramine and colestipol are resins that exchange anions and form complexes with organic ones like bile acids with high affinity. The main disadvantage of cholestyramine is that it possesses an unpleasant taste, which can result in poor patient compliance. It is usually well tolerated but can cause constipation. Other side effects, which can present in up to $30 \%$ of patients treated with cholestyramine, include flatulence, borborygmi, abdominal pain and nausea. Both cholestyramine and colestipol can interact with other drugs such as thiazide diuretics, digoxin, beta-blockers and thyroid hormones, reducing their absorption and serum concentrations. Therefore, it is recommended for these drugs to be administered 2 hours before the bile acid binders. Studies have suggested a doseresponse relationship according to severity of diarrhea, based upon the results of the SeHCAT: response to cholestyramine was detected in $96 \%$ of patients with $<5 \%$ retention in the SeHCAT, $80 \%$ at $<10 \%$ and $70 \%$ at $<15 \%$.

Colesevelam is the newest bile acid binder. It has a higher affinity for bile acids than cholestyramine or colestipol and since its presentation is in tablets, it does not have an unpleasant taste, which is beneficial for patient compliance. Colesevelam is useful in patients who have undergone treatment with cholestyramine but it wasn't successful.

\section{Summary and recommendations}

Bile acid malabsorption is a frequently ignored differential diagnosis in patients with chronic diarrhea. Even though it is not an uncommon condition, gastroenterologists fail to assess their patients for it. BAM should always be suspected in patients with chronic, watery diarrhea, particularly in patients with history of ileal disease, bowel resection due to Crohn's disease or other related gastrointestinal pathologies. Type 2 BAM or idiopathic BAM, can also be a contributing factor in causing diarrhea in one-third of patients with IBS-D. The etiology of idiopathic BAM is still not entirely understood but it is believed alterations in the enterohepatic circulation, lowered FGF19 levels.

Since diagnostic tests like SeHCAT or C4 are not readily available in all medical centers and gastroenterology practices, it is recommended to provide an empirical treatment with bile acid binders, such as cholestyramine, in case BAM is the cause of chronic diarrhea, as currently recommended by American Gastroenterological Association and British Society of Gastroenterology guidelines.

\section{Acknowledgements}

Jose Nieto: Wrote the manuscript, researched the literature and is the article guarantor.

Amanda Bastidas: Wrote the manuscript and researched the literature.

\section{Conflicts of interest}

The authors have no conflict of interest for this research.

\section{Funding}

None.

\section{References}

1. Barkun A, Love J, Gould M, et al Bile acid malabsorption in chronic diarrhea: pathophysiology and treatment. Can J Gastroenterol. 2013;27(11):653-659.

2. Khalid U, Lalji A, Stafferton R, et al. Bile acid malabsoption: a forgotten diagnosis?. Clin Med (Lond). 2010;10(2):124-126.

3. Small D, Dowling RH, Redinger RN. The enterohepatic circulation of bile salts. Arch Intern Med. 1972;130(4): 552-573.

4. Camilleri M. Bile Acid Diarrhea: prevalence, pathogenesis, and therapy. Gut Liver. 2015;9(3):332-339.

5. Swell L, Gustafsson J, Schwartz C, et al. An in vivo evaluation of the quantitative significance of several potential pathways to cholic and chenodeoxycholic acids from cholesterol in man. $J$ Lipid Res. 1980;21(4):455-466.

6. Chiang J. Regulation of synthesis. J Lipid Res. 2009;50(10):1955-1966.

7. Mekhjian H, Phillips S, Hofmann AF. Colonic absorption of unconjugated bile acids: perfusion studies in man. Dig Dis Sci. 1979;24(7):545-550.

8. Walters JN, Pattni S. Recent advances in the understanding of bile acid malabsorption. Br Med Bull. 2009;92:79-93. 